\title{
Establishment of a Method to Allow Microbial Limit Testing of lodine-containing Preparations
}

\author{
Huang Minna, Tan Fangming, Lin Shaozhu, Liang Zhuanzhi, Qiu Suishan, He Xiaomin* \\ Department of Pharmacy, The First Affiliated Hospital of Jinan University, Guangzhou, China \\ Email address: \\ 418553103@qq.com (He Xiaomin) \\ ${ }^{*}$ Corresponding author \\ To cite this article: \\ Huang Minna, Tan Fangming, Lin Shaozhu, Liang Zhuanzhi, Qiu Suishan, He Xiaomin. Establishment of a Method to Allow Microbial Limit \\ Testing of Iodine-containing Preparations. Journal of Drug Design and Medicinal Chemistry. Vol. 7, No. 2, 2021, pp. 39-43. \\ doi: $10.11648 /$ j.jddmc.20210702.12
}

Received: April 22, 2021; Accepted: May 17, 2021; Published: May 26, 2021

\begin{abstract}
Background: Contamination of pharmaceuticals with microorganisms may lead to deleterious effects on the therapeutic properties of the drug, and may potentially cause injuries to intended recipients. Iodine can inhibit and kill microorganisms in pharmaceutical preparations. The risk of microbial contamination of cellular products can be reduced when cultured in the presence of iodine. This however, may impact the sensitivity of microbiological tests. Iodine-containing Preparations does not guarantee sterility but may just reduce the proliferation rate of microorganisms, microbiological testing of medicinal products remains obligatory. Thus, an appropriate method to test for microbial contamination of iodine-containing products has to be validated. Objective: To establish a method that would allow microbial limit testing of four iodine-containing preparations (concentrated iodine tincture, compound iodine oral solution, iodine tincture, and iodine glycerin). Methods: To specifically determine the degree of contamination in the four in-house iodine preparations, sodium thiosulfate was used to reduce the iodine molecules to iodide ions, thereby eliminating the intrinsic antibacterial property of the preparations. Then, the suitability of this method was evaluated according to the regulations for non-sterile microbial limit tests. Results: The neutralization method effectively eliminated the interference of the antibacterial iodine component, rendering the control bacteria detectable. The recovery ratio of the test strains met the required standards. Conclusion: The neutralization method with sodium thiosulfate as the iodine neutralizer is suitable for microbial limit testing of concentrated iodine tincture, compound iodine oral solution, iodine tincture, and iodine glycerin, with the results being accurate and reliable.
\end{abstract}

Keywords: Iodine-containing Preparations, Microbial Limit Test, Neutralization Method, Verification

\section{Introduction}

The microbial contamination of drugs not only affects the curative effects of the medication directly but can also endanger the health and life of the medication user. Therefore, microbial control is an important part of drug quality testing [1, 2]. Concentrated iodine tincture, compound iodine oral solution, iodine tincture, and iodine glycerin are four in-house preparations developed by the First Affiliated Hospital of Jinan University that have been used clinically for many years with good safety and definite curative effects. The four preparations are composed mainly of iodine and potassium iodide. The content of iodine is $10 \%, 5 \%, 3 \%$, and $2 \%$ respectively. Concentrated iodine tincture, iodine tincture, and iodine glycerin are disinfectants and antiseptics. Concentrated iodine tincture is used to treat onychomycosis; iodine tincture is used for skin infection and disinfection; iodine glycerin is used for oral mucosal infection. The compound iodine oral solution supplements iodine, regulates thyroid function, and is used for treatment of simple goiter and preoperative preparation before hyperthyroidism surgery. Iodine can inhibit and kill microorganisms in pharmaceutical preparations. Free iodine penetrates the bacterial cell wall easily and combines with the hydroxyl, hydrocarbon, and sulfhydryl groups on the amino acid chains of proteins to cause their denaturation and precipitation, thereby rendering them halogenated so that they lose their biological activity [3-6]. However, there is no standardized method for microbial limit testing of iodine-containing solutions, and thus, we are unable to accurately determine whether in-house iodine preparations are contaminated by microorganisms. Therefore, this study was 
carried out to establish such a test method for the four in-house preparations. To truly reflect the degree of contamination of the preparations, sodium thiosulfate was used to reduce the iodine molecules to iodide ions, thereby eliminating the intrinsic antibacterial property of the iodine preparations. Then, following addition of several test microorganisms to the neutralizer-treated preparations, standard plate-counting and bacterial control methods were carried out to determine if the test microbial species could still be detected accurately and reliably.

\section{Materials and Methods}

\subsection{Samples}

The concentrated iodine tincture, compound iodine oral solution, iodine tincture, and iodine glycerin are all in-house preparations of the First Affiliated Hospital of Jinan University. Three sample batches were used for each preparation.

\subsection{Bacterial Test Strains, Culture Media, and Chemical Reagent}

Staphylococcus aureus, Bacillus subtilis, Pseudomonas aeruginosa, Candida albicans, Aspergillus niger, and Escherichia coli were provided by the Guangdong Province Institute for Drug Control and Guangdong Microbial Culture Collection Center (Guangdong, China). These test strains were used at passage numbers 3-4. The tryptic soy peptone agar medium, tryptic soy peptone liquid medium (broth), Sabouraud dextrose agar medium, Sabouraud dextrose broth, sodium chloride-peptone buffer solution ( $\mathrm{pH}$ 7.0), cetyltrimethylammonium bromide agar medium, mannitol salt agar medium, MacConkey broth, and MacConkey agar medium were all produced by Guangdong Huankai Microbial Technology Co., Ltd (Guangdong, China). Sodium thiosulfate was analytically pure, which produced by Tianjin Damao Chemical Reagent Factory.

\subsection{Test Methods}

The suitability of the method for microbial limit testing was determined according to the standards of the "Chinese Pharmacopoeia, 2015 edition," mainly by plate counting of the total number of aerobic bacteria and the total number of molds and yeasts and by testing for the control bacteria [7]. These three independent tests were performed in parallel. The recovery ratios of the test bacteria were calculated, and the ability to detect the relevant test strain was determined with the control bacteria test.

\subsection{Preparation of the Bacterial Samples}

Staphylococcus aureus, Pseudomonas aeruginosa, and Bacillus subtilis were respectively inoculated into tryptic soy peptone broth and cultured at $35^{\circ} \mathrm{C}$ for $24 \mathrm{~h}$. Candida albicans was inoculated into Sabouraud dextrose broth, cultured at $25^{\circ} \mathrm{C}$ for $48 \mathrm{~h}$, and then diluted with sterile sodium chloride solution to form the yeast solution for later use. Aspergillus niger was inoculated on Sabouraud dextrose agar medium and cultured for
5 days at $25^{\circ} \mathrm{C}$. Then, $5 \mathrm{~mL}$ of sterile sodium chloride-peptone buffer ( $\mathrm{pH} 7.0$ ) containing $0.05 \%$ (v/v) polysorbate 80 was added to elute the spores. The spore suspension was then aspirated to a sterile test tube and diluted with sterile sodium chloride-peptone buffer ( $\mathrm{pH} 7.0$ ) containing $0.05 \%$ polysorbate 80 to form the mold solution for later use [7].

\subsection{Preparation of the Test Solutions}

In brief, $10 \mathrm{~mL}$ each of concentrated iodine tincture, compound iodine oral solution, iodine tincture, and iodine glycerin was respectively added to $90 \mathrm{~mL}$ of sterile sodium chloride-peptone buffer ( $\mathrm{pH}$ 7.0) containing 2.2, 1.1, 0.7, or0.5 $\mathrm{g}$ of sodium thiosulfate.

\subsection{Plate Counting of the Total Number of Aerobic Bacteria and the Total Number of Molds and Yeasts}

Test group: Dry sterile test tubes, each containing $9.9 \mathrm{~mL}$ of the individual test solutions, were first set up. Then, $0.1 \mathrm{~mL}$ each of the Staphylococcus aureus, Pseudomonas aeruginosa, Bacillus subtilis, Candida albicans, and Aspergillus niger preparations containing no more than $10^{4} \mathrm{CFU}$ of the microbial strains was added to the test tubes. After thorough mixing, $1 \mathrm{~mL}$ of the resulting bacterial solutions were added to sterile $90 \mathrm{~mm}$ petri dishes, then overlaid with $20 \mathrm{~mL}$ of tryptic soy peptone agar medium, and thereafter cultured at $35^{\circ} \mathrm{C}$ for 48 h. For the Candida albicans and Aspergillus niger solutions, $3.3 \mathrm{~mL}$ each was added to sterile $150 \mathrm{~mm}$ petri dishes, then overlaid with $50 \mathrm{~mL}$ of Sabouraud dextrose agar medium, and subsequently cultured for 5 days at $25^{\circ} \mathrm{C}$. Two petri dishes were prepared in parallel for each test solution and test strain combination, and the number of colonies on each dish was counted, with results given as the average number of colonies.

Test sample control group: The test solutions without any test strains were mixed with the neutralizer and spread onto the various plates, using the same protocol as used for the test groups, and the number of colonies on the plates was calculated.

Bacteria solution group: In brief, $0.1 \mathrm{~mL}$ each of the Staphylococcus aureus, Pseudomonas aeruginosa, Bacillus subtilis, Candida albicans, and Aspergillus niger preparations containing not more than $10^{4} \mathrm{CFU}$ of bacterial strains was respectively added to $9.9 \mathrm{~mL}$ of aseptic sodium chloride-peptone buffer ( $\mathrm{pH}$ 7.0). After thorough mixing, the solutions were spread onto the various plates, using the same protocols as used for the test groups, and the number of colonies in the bacterial solution group was calculated.

Neutralizer control group: In brief, $2.2 \mathrm{~g}$ of sodium thiosulfate was added to $100 \mathrm{~mL}$ of sterile sodium chloride-peptone buffer $(\mathrm{pH} 7.0)$ and then spread onto the various plates together with the test strains, as described for the test groups. The number of colonies in the control solution was then calculated.

Calculation of the recovery ratios: The recovery ratio of the test group $=[$ (average colony count of the test group - average colony count of the test solution group)/average colony count of the bacterial solution group]; the recovery ratio of the neutralizer control group $=$ the average colony count of the neutralizer control group/the average colony count in the 
bacterial solution group.

\subsection{Adaptability of the Method to the Control Bacteria Test}

According to the requirements of the 2015 edition of "Chinese Pharmacopoeia," in the test of control bacteria, compound iodine oral solution is an oral liquid preparation for which the presence of Escherichia coli must be tested; iodine tincture and concentrated iodine tincture are skin administration preparations for which Staphylococcus aureus and Pseudomonas aeruginosa must be tested; and iodine glycerin is an oral mucosal administration preparation for which Escherichia coli, Staphylococcus aureus, and Pseudomonas aeruginosa must be tested.

Test group: Ten milliliters each of the test solutions was inoculated into $100 \mathrm{~mL}$ of tryptic soy peptone broth. Then, 50-100 CFU of Escherichia coli, Staphylococcus aureus, and Pseudomonas aeruginosa was respectively added to the solutions for culture at $35^{\circ} \mathrm{C}$ for $24 \mathrm{~h}$. Thereafter, $1 \mathrm{~mL}$ of the Escherichia coli-containing broths was respectively inoculated into $100 \mathrm{~mL}$ of MacConkey broth and the cultures were incubated for $48 \mathrm{~h}$ at $42^{\circ} \mathrm{C}$. These cultures were then streaked onto MacConkey agar medium plates and incubated for $72 \mathrm{~h}$ at $35^{\circ} \mathrm{C}$. The Staphylococcus aureus-containing broths were streaked onto mannitol salt agar medium plates and incubated for $48 \mathrm{~h}$ at $35^{\circ} \mathrm{C}$. The Pseudomonas aeruginosa-containing broths were streaked onto cetyltrimethylammonium bromide agar medium plates and incubated for $24 \mathrm{~h}$ at $35^{\circ} \mathrm{C}$.

Test solution control group: Ten milliliters each of the test solutions was inoculated into $100 \mathrm{~mL}$ of tryptic soy peptone broth. No test bacteria were added. The mixtures were then processed in the same way as carried out for the test group.

Negative control group: $10 \mathrm{~mL}$ of sterile sodium chloride-peptone buffer ( $\mathrm{pH}$ 7.0) was inoculated into $100 \mathrm{~mL}$ of tryptic soy peptone broth. No test bacteria were added. The mixtures were then processed in the same way as carried out for the test group.

Positive control group: $10 \mathrm{~mL}$ of sterile sodium chloride-peptone buffer ( $\mathrm{pH}$ 7.0) was inoculated into $100 \mathrm{~mL}$ of tryptic soy peptone broth. Then, the Escherichia coli, Staphylococcus aureus, and Pseudomonas aeruginosa preparations containing 50-100 CFU of the bacterial strains were added, respectively. The mixtures were then processed in the same way as carried out for the test group.

Neutralizer control group: In brief, $2.2 \mathrm{~g}$ of sodium thiosulfate was added to $100 \mathrm{~mL}$ of sterile sodium chloride-peptone buffer ( $\mathrm{pH} 7.0$ ) and $10 \mathrm{~mL}$ of this mixture was then inoculated into $100 \mathrm{~mL}$ of tryptic soy peptone broth. Thereafter, the Escherichia coli, Staphylococcus aureus, and Pseudomonas aeruginosa preparations containing 50-100 CFU of the bacterial strains were added, respectively. The bacterial mixtures were then processed in the same way as carried out for the test group.

\section{Results}

In the test for counting the total number of aerobic bacteria and total number of molds and yeasts, the recovery ratios of the test group were in the range of $0.5-2$ (Tables 1 and 2).

Table 1. Recovery ratios of the test bacteria using the total aerobic bacteria plate count method.

\begin{tabular}{|c|c|c|c|c|c|}
\hline \multirow{2}{*}{ Sample name } & \multicolumn{5}{|c|}{ Average recovery ratios of the test strains } \\
\hline & Staphylococcus aureus & Pseudomonas aeruginosa & Bacillus subtilis & Candida albicans & Aspergillus niger \\
\hline Compound iodine oral solution & 0.7 & 0.9 & 1.0 & 1.0 & 1.0 \\
\hline Iodine tincture & 1.0 & 0.9 & 1.2 & 1.0 & 0.8 \\
\hline Concentrated iodine tincture & 0.9 & 0.8 & 1.2 & 0.8 & 0.8 \\
\hline Iodine glycerin & 1.0 & 0.9 & 1.0 & 0.9 & 0.9 \\
\hline Neutralizer control group & 0.8 & 0.8 & 1.1 & 0.9 & 0.9 \\
\hline
\end{tabular}

Table 2. Recovery ratios of the test yeast and mold using the total plate count method.

\begin{tabular}{lll}
\hline \multirow{2}{*}{ Sample name } & \multicolumn{2}{l}{ Average recovery ratios of test strains } \\
\cline { 2 - 3 } & Candida albicans & Aspergillus niger \\
\hline Concentrated iodine tincture & 0.9 & 0.8 \\
Compound iodine oral solution & 0.9 & 0.9 \\
Iodine tincture & 0.9 & 0.8 \\
Iodine glycerin & 1.1 & 0.8 \\
Neutralizer control group & 1.2 & 0.9 \\
\hline
\end{tabular}

Moreover, in the control bacteria test, the test solutions were positive for their respective control bacteria, and the bacterial numbers in the other control groups met the national requirements. The control bacteria in the test group, the positive control group, and the neutralizer control group grew well (Table 3). Moreover, no colony growth was seen in the test solution control group and the negative control group (Table 3).

Table 3. Results of the applicability of the method for the control bacteria test.

\begin{tabular}{|c|c|c|c|c|c|c|}
\hline Sample name & Test strains & $\begin{array}{l}\text { Test } \\
\text { group }\end{array}$ & $\begin{array}{l}\text { Test solution } \\
\text { control group }\end{array}$ & $\begin{array}{l}\text { Negative control } \\
\text { group }\end{array}$ & $\begin{array}{l}\text { Positive control } \\
\text { group }\end{array}$ & $\begin{array}{l}\text { Neutralizer } \\
\text { control group }\end{array}$ \\
\hline \multirow{2}{*}{ Concentrated iodine tincture } & Staphylococcus aureus & + & - & - & + & + \\
\hline & Pseudomonas aeruginosa & + & - & - & + & + \\
\hline Compound iodine oral solution & Escherichia coli & + & - & - & + & + \\
\hline
\end{tabular}




\begin{tabular}{|c|c|c|c|c|c|c|}
\hline Sample name & Test strains & $\begin{array}{l}\text { Test } \\
\text { group }\end{array}$ & $\begin{array}{l}\text { Test solution } \\
\text { control group } \\
\end{array}$ & $\begin{array}{l}\text { Negative control } \\
\text { group }\end{array}$ & $\begin{array}{l}\text { Positive control } \\
\text { group }\end{array}$ & $\begin{array}{l}\text { Neutralizer } \\
\text { control group }\end{array}$ \\
\hline \multirow{2}{*}{ Iodine tincture } & Staphylococcus aureus & + & - & - & + & + \\
\hline & Pseudomonas aeruginosa & + & - & - & + & + \\
\hline \multirow{3}{*}{ Iodine glycerin } & Escherichia coli & + & - & - & + & + \\
\hline & Staphylococcus aureus & + & - & - & + & + \\
\hline & Pseudomonas aeruginosa & + & - & - & + & + \\
\hline
\end{tabular}

Remarks: "+" means that a positive strain was detected; "-" means that no strain was detected.

The results indicated that the use of sodium thiosulfate as a neutralizer could effectively eliminate interference from the inhibitory iodine component in various microbial limit tests. Additionally, the neutralizer was non-toxic to the test bacteria, making it suitable for microbial limit testing of the concentrated iodine tincture, compound iodine oral solution, iodine tincture, and iodine glycerin.

\section{Discussion}

Iodine has powerful disinfectant and antiseptic effects and can kill most bacteria and bacterial spores, viruses, and fungi. Previous uses of the dilution method, polysorbate 80 neutralization method, and membrane filtration method failed to effectively eliminate the antibacterial effects of iodine [8]. Therefore, in the present study, the neutralizer sodium thiosulfate was used to eliminate the antibacterial effect of elemental iodine via the oxidation-reduction reaction between the two. The amount of sodium thiosulfate required for neutralization is calculated according to following the chemical equation:

$$
\mathrm{I}_{2}+2 \mathrm{Na}_{2} \mathrm{~S}_{4} \mathrm{O}_{6}=\mathrm{Na}_{2} \mathrm{~S}_{4} \mathrm{O}_{6}+2 \mathrm{NaI}
$$

According to the equation, $1 \mathrm{~mol}$ of iodine is equivalent to 1 mol of sodium thiosulfate [9]; that is, the amount of sodium thiosulfate needed to neutralize $10 \mathrm{~mL}$ each of concentrated iodine tincture, compound iodine oral solution, iodine tincture, and iodine glycerin is $1.96 \mathrm{~g}, 0.98 \mathrm{~g}, 0.59 \mathrm{~g}$, and $0.39 \mathrm{~g}$, respectively. Because the concentration range of the four preparations is $90-110 \%$ of the labeled amount, the amount of sodium thiosulfate used has to be slightly larger than the actual mass after conversion in order to ensure the complete elimination of the antibacterial effect of iodine.

The test results show that adding a certain amount of sodium thiosulfate can effectively eliminate the antibacterial properties of iodine, and the neutralizer has no effect on the detection of test bacteria. This method is simple and effective, ensuring the scientific accuracy and reliability of microbial limit test results of concentrated iodine tincture, compound iodine oral solution, iodine tincture, and iodine glycerin [10-15].

\section{Conclusion}

In conclusion, this microbial limit test method control and ensure the quality of drug production by solving the problem of interference of antimicrobial components in drugs. It can eliminate the bacteriostasis of iodine.

\section{References}

[1] R. Zhu, H. y. Xu, Z. H. Song. Review on Revision of the Standards System for Pharmaceutical Microbial Control of the Chinese Pharma-copoeia 2020 Edition Volume IV [J]. Chinese Pharmac- eutical Journal, 2020, 55 (19): 1564-1568.

[2] W. Y. Zhang, F. Q. Guo. Results of Microbial Limit Tests and Quality Analysis of Hospital Preparation Sampling Inspection of Tianjin [J]. Chinese Pharmaceutical Affairs, 2019, 33 (01): 100-107.

[3] S. F. Cai, J. L. Li, X. Xu, J. S. Huang. Study on Microbial Limit Test for Hospital Preparation of Onych-omycosis [J]. Strait Pharmaceutical Journal, 2021, 33 (01): 72-75.

[4] Y. Zhao, C. Shi, P. Huang. Methodology Validation of Microbial Limit Test for Iodine tincture [J]. China Pharmacist, 2011, 14 (9): 1382-1383.

[5] Cooper RA. Iodine revisited [J]. International Wound Journal, 2007, 4 (2): 124-37.

[6] J. Li, H. Cai, H. M. Cao, Q. He, Establishment of Microbial Limit Test for Iodine Glycerin [J]. China Pharmaceuticals, 2019, 28 (27): 30-33.

[7] ChP 2015. Vol IV [S]. 2015: Appendix 1105, 1106.

[8] J. Ji, T. T. Liu, M. M. Niu, Z. W. Wang. Neutralization of Lecithin and Polysorbate- 80 in Antibacterial Activity of Traditional Chinese Medicine Preparations [J]. China Pharmacist, 2017, 20 (3): 586-588.

[9] L. N. Li. Methodology Validation of Microbial Limit Test for compound iodine oral solution [J]. Inner Mongolia Journal of Traditional Chinese Medicine, 2016, 10 (13): 143-144.

[10] Sh. Ma, P. Liu, L. H. Yang, et al. Experimental study on microorganism introduction method in suitability test of microbial limit test [J]. Chin J Pharm Anal, 2018, 38 (5): 878-882.

[11] X. L. Yang, H. Li, Y. Y. Ma, et al. Interpretation of the microbio-logical examination of nonsterile products: microbial enumeration test of Chinese pharmacopoeia 2015 edition [J]. Chin J Pharm A- nal, 2016, 36 (6): 1101-1107.

[12] X. L. Yang, H. Li, Y. Y. Ma, et al. Interpretation and Countermea-sure of the Microbiological Examination for Nonsterile Products: Tests for Specified Microorganisms in Chinese Pharma- copoeia 2015 Edition [J]. China Pharm, 2016, 19 (4): 748-752.

[13] Y. J. Pang, W. Y. Fan, J. Liang. Research progress on methodological validation of microbial limit test for pharmaceuticals [J]. Chinese Traditional Patent Medicine, 2017, 39 (10): 2137-2140. 
[14] H. L. Ma, Y. Y. Tao, Ch. F. Xu, Y. M. Zhang. Study on applicability of microbial limit test methods for three kinds of hospital preparations [J]. International Journal of Traditional Chinese Medicine, 2020, 42 (5): 469-474.
[15] W. Liu, G. Q. Zhang, L. G. Zhu. Establishment of a Microbial Limit Test Method for Two Iodine Preparations in Chinese Pharmacopoeia (2015 Edition) [J]. Chinese Journal of Rational Drug Use, 2017, 14 (2): 14-17. 